\begin{tabular}{|c|l|}
\hline Title & Optical observation of superconducting density of states in luminescence spectra of InA s quantum dots \\
\hline Author(s) & Mou, S. S.; Irie, H.; A sano, Y.; A kahane, K.; Nakajima, H.; Kumano, H.; Sasaki, M.; Muray ama, A .; Suemune, I. \\
\hline Citation & $\begin{array}{l}\text { Physical review B, 92(3), 035308-1-035308-9 } \\
\text { https://doi.org/10.1103/PhysRevB.92.035308 }\end{array}$ \\
\hline Issue Date & 2015-07-15 \\
\hline Doc URL & http://hdl.handle.net/2115/59814 \\
\hline Rights & @2015A American Physical Society \\
\hline Type & article \\
\hline File Information & PhysRevB.92.035308.pdf \\
\hline
\end{tabular}

Instructions for use 


\title{
Optical observation of superconducting density of states in luminescence spectra of InAs quantum dots
}

\author{
S. S. Mou, ${ }^{1}$ H. Irie, ${ }^{2}$ Y. Asano, ${ }^{3}$ K. Akahane, ${ }^{4}$ H. Nakajima, ${ }^{1}$ H. Kumano, ${ }^{1}$ M. Sasaki,${ }^{4}$ A. Murayama, ${ }^{5}$ and I. Suemune ${ }^{1, *}$ \\ ${ }^{1}$ Research Institute for Electronic Science, Hokkaido University, Sapporo 001-0020, Japan \\ ${ }^{2}$ NTT Basic Research Laboratories, NTT Corporation, Atsugi 243-0198, Japan \\ ${ }^{3}$ Graduate School of Engineering, Hokkaido University, Sapporo 060-8628, Japan \\ ${ }^{4}$ National Institute of Information and Communication Technology, Koganei 184-8795, Japan \\ ${ }^{5}$ Graduate School of Information Science and Technology, Hokkaido University, Sapporo 060-0814, Japan
}

(Received 31 May 2015; revised manuscript received 11 July 2015; published 27 July 2015)

\begin{abstract}
We study luminescence spectra observed from InAs quantum dots (QDs) embedded in an $n$-type InGaAs-based heterostructure, where electron Cooper pairs penetrate from an adjacent niobium $(\mathrm{Nb})$ superconductor with the proximity effect. Below the superconducting (SC) critical temperature of $\mathrm{Nb}$, we observe substantial luminescence intensity enhancement and a sharp edge in luminescence spectra of InAs QDs. We explain the observed sharp edge in the luminescence spectra with the proximity effect, that is, with the consideration of opening of SC gap and modification of density of states (DOS) near the electron Fermi level in the $n$-type semiconductor heterostructure. We demonstrate that the sharp edge luminescence spectra are well reproduced by the SC DOS, with quasiparticle lifetime broadening and a Gaussian distribution of lowest QD state emission lines. We discuss the reason why it has been difficult to observe the sharp edge luminescence spectra in the previous quantum well-based SC light emitting diodes.
\end{abstract}

DOI: 10.1103/PhysRevB.92.035308

PACS number(s): 74.25.Gz, 74.25.Jb, 74.45.+c, 78.55.-m

\section{INTRODUCTION}

Quantum information and communications technologies are expected to open the way for realizing efficient information processing [1] and secure information transmission [2]. The quantum entanglement plays an essential role in quantum information processing tasks, such as quantum teleportation [3-5] and entanglement swapping [6-8]. Parametric down conversion (PDC) has been widely used for quantum entangled photon pairs (QEPPs) sources [9], and the QEPP generation rate has recently been dramatically improved [10]. The intrinsic drawbacks of PDC are the nonzero probability of multiple photon emission at a time and nondeterministic nature of generation processes.

Solid-state sources have been actively studied for realizing on-demand operations with minimum multiple-photon generation probability. The method most frequently used is the biexciton-exciton cascaded emission of quantum dots (QDs) [11-13]. The violation of Bell's inequality, which quantifies the high degree of entanglement [14], was demonstrated by a QD grown on a GaAs (001) substrate with temporal postselection to reduce temporal gates [15]. It was also demonstrated by an isotropic QD grown with droplet epitaxy on a GaAs (111)A substrate [16]. However, demonstration of such a performance still remains difficult for most QDs prepared with normal methods, due to the fine structure splitting (FSS) of the exciton states, which is induced mainly by their structural anisotropy [17]. Photons emitted from the biexciton-exciton cascade become energetically distinguishable by the FSS of the intermediate exciton states, and this prevents their entanglement.

Instead of the cascaded photon emission, simultaneous twophoton emission (TPE) is preferable from the viewpoint of the

\footnotetext{
*isuemune@es.hokudai.ac.jp
}

little relative time delay and relative time jitter of the two photons for operations such as entanglement swapping. The TPE has been observed from atoms, such as hydrogen [18], but the emission rate is extremely low. It is also possible with semiconductors, but their TPE probability is five orders of magnitude weaker than the competitive one-photon emission [19], and the efficiency is quite low.

We proposed to generate QEPP by converting electron Cooper pairs, which form spin-singlet pairs and are entangled electron pairs [20], to photon pairs through radiative recombination with pairs of holes [21]. We fabricated a superconducting (SC) light emitting diode (LED), where the $n$-type electrode was replaced with a niobium $(\mathrm{Nb}) \mathrm{SC}$ electrode. We observed enhancement of luminescence at the wavelength of $\sim 1.6 \mu \mathrm{m}$ below the $\mathrm{SC}$ critical temperature $\left(T_{\mathrm{C}}\right)$ of $\mathrm{Nb}$ [22]. We also observed the reduction of luminescence decay time constant below $T_{\mathrm{C}}$, which is the indication of the enhanced photon emission rate [23].

The key issue of the SC LEDs is the proximity effect [24], where a normal metal in close proximity to a superconductor acquires SC properties, and its local density of states (DOS) is modified by the penetration of electron Cooper pairs. Experimentally, a normal metal surface that includes several SC islands was studied with scanning tunneling spectroscopy (STS) [25], and the proximity effect, especially spatial extension of the SC properties from the superconductor interface to the normal metal surface, was directly observed. Opening of the SC gap and the modified SC DOS were observed on the normal-metal surface adjacent to the SC-island interface. Also, opening of the SC gap and the SC DOS induced in a semiconductor by the proximity effect was observed with InAsaluminum $(\mathrm{Al})$ semiconductor-superconductor nanowires with tunneling spectroscopy [26].

We have analyzed the Cooper-pair luminescence based on the second-order perturbation theory for the electronphoton interaction [27]. The superconductivity effect was 
included through the Bogoliubov transformation from electron operators to Bogoliubov quasiparticle operators [28]. This theory predicted luminescence enhancement near the SC DOS. Although we observed the predicted luminescence intensity enhancement, we could not observe any distinct variation of the luminescence spectra emitted from quantum well (QW) active layers of the SC LEDs [22,23,29,30].

Our original proposal of the SC LED was based on a QD active layer [21]. When electrons near the electron Fermi level form spin-singlet Cooper pairs, they change from fermions to bosons of which number states are not fixed due to their coherent nature [30]. The photon emission from the SC LED is regulated by minority holes populating the lowest-energy QD state in the valence band, which is under the Pauli's exclusion principle. We have recently studied luminescence spectra of InAs QDs that are in proximity to a $\mathrm{Nb}$ superconductor and reported preliminary observation of anomalous luminescence spectra below $T_{\mathrm{C}}$ of $\mathrm{Nb}$ [31].

In this paper, we study the InAs QD-based semiconductor/ $\mathrm{Nb}$ superconductor system in detail. We observe a sharp edge in luminescence spectra of InAs QDs, as well as the luminescence intensity enhancement below $T_{\mathrm{C}}$ of $\mathrm{Nb}$. We study the excitation power dependence of the observed sharp edge and explain our observation with the proximity effect, considering the opening of SC gap and the SC DOS in the $n$-type semiconductor heterostructure, in which the QDs are embedded. We also discuss the reason why the variation of luminescence spectra has not been observed in the previous QW-based SC LEDs below and above $T_{\mathrm{C}}$ of $\mathrm{Nb}$.

\section{SAMPLE PREPARATION AND MEASUREMENT SETUP}

For practical applications, a LED structure is most suitable for driving photon sources by electric current. However, in the InAs QD-based semiconductor/Nb superconductor system, a $p$ - $n$ junction induces the internal built-in potential that is varied with external bias. The depletion layer induced at the junction by the built-in potential prevents penetration of Cooper pairs into QDs located at the junction. Therefore, application of external bias changes both the potential profile near QDs and the current injection, and this makes the situation complex. In this regard, uniform $n$-type doping keeps the electron Fermi level constant in the whole semiconductor structure and offers simpler basis for the study of the proximity effect. Then, by injecting minority holes with an external optical excitation, we can produce supercurrent from the adjacent superconductor to InAs QDs in the event of Cooper-pair luminescence.

Based on this consideration, a QD-based heterostructure was designed, as shown in Fig. 1(a) and was fabricated with molecular beam epitaxy (MBE) on an InP (311)B substrate. The reason for the growth on the $\operatorname{InP}(311) \mathrm{B}$ substrate is that growth on usual InP (001) substrates results in elongated quantum dashes rather than QDs [32]. The undoped $50 \mathrm{~nm}$ thick $\mathrm{In}_{0.53} \mathrm{Ga}_{0.47}$ As and $100 \mathrm{~nm}$ thick $\operatorname{In}_{0.52} \mathrm{Al}_{0.48}$ As layers were grown for removing the InP substrate with selective chemical etching. Then, the $200 \mathrm{~nm}$ thick $n$-type $\mathrm{In}_{0.53} \mathrm{Ga}_{0.25} \mathrm{Al}_{0.22} \mathrm{As}$ and $20 \mathrm{~nm}$ thick $n$-type $\operatorname{In}_{0.53} \mathrm{Ga}_{0.35} \mathrm{Al}_{0.12} \mathrm{As}$ barrier layers were grown. All the above layers were grown at $470{ }^{\circ} \mathrm{C}$. Then, the five monolayers (ML) of undoped InAs QDs were grown at the higher temperature of $530^{\circ} \mathrm{C}$. The QDs grown

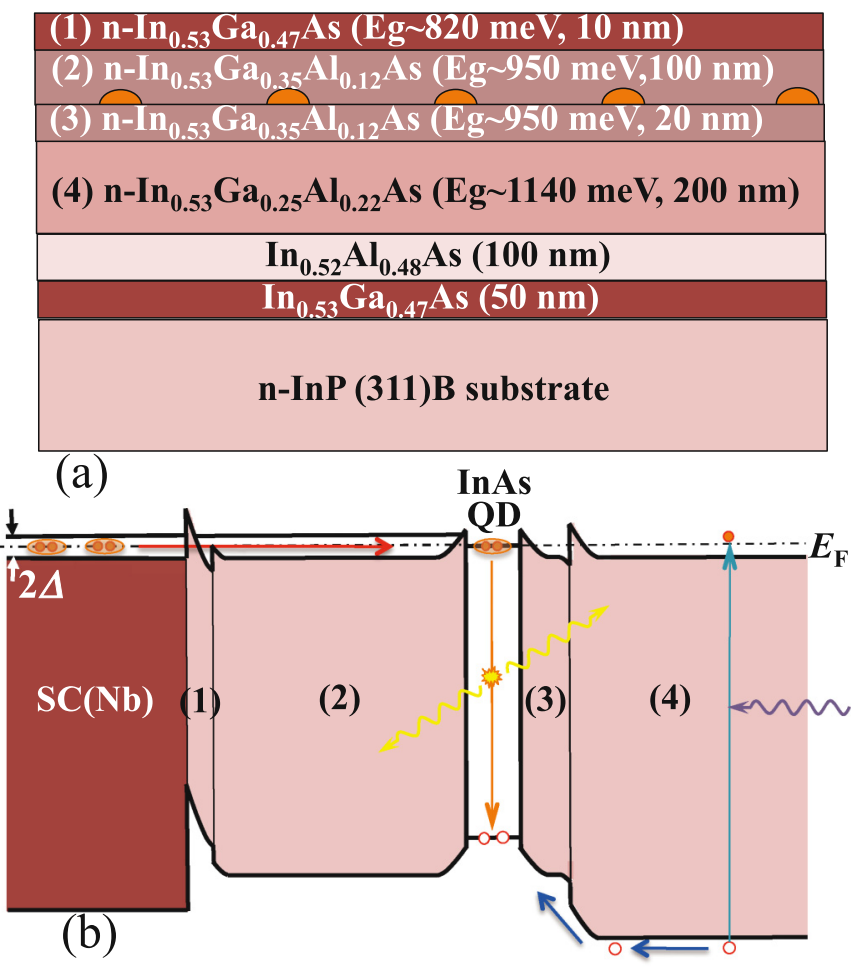

FIG. 1. (Color online) (a) Schematic of InAs QD-based heterostructure sample. (b) Schematic band structure after the $\mathrm{Nb}$ deposition on the sample surface. The layers (1)-(4) correspond to those shown in (a). Below $T_{\mathrm{C}}$, the $\mathrm{SC}$ gap $2 \Delta$ opens in the $\mathrm{Nb}$ superconductor, and Cooper pairs penetrate into InAs QDs through the semiconductor heterostructure with the proximity effect. Minority holes are generated with the external $\mathrm{He}-\mathrm{Ne}$ laser irradiation and transported to InAs QDs. A Cooper pair recombines with a pair of holes and generates a pair of photons.

on the (311)B crystal surface keep a circular shape, but the QD density is high, typically $9 \times 10^{10} \mathrm{~cm}^{-2}$, at $470^{\circ} \mathrm{C}$. It is reduced to the density of $3.4 \times 10^{10} \mathrm{~cm}^{-2}$ at $530^{\circ} \mathrm{C}$ [33]. The average QD lateral size and height are 57 and $5.6 \mathrm{~nm}$, respectively. After the QDs growth, the substrate temperature was lowered to $470{ }^{\circ} \mathrm{C}$ again, and the subsequent $100 \mathrm{~nm}$ thick $n$-type $\operatorname{In}_{0.53} \mathrm{Ga}_{0.35} \mathrm{Al}_{0.12} \mathrm{As}$ barrier and $10 \mathrm{~nm}$ thick $n$-type $\mathrm{In}_{0.53} \mathrm{Ga}_{0.47}$ As contact layers were grown. The $n$-type doping level was designed to be $1 \times 10^{18} \mathrm{~cm}^{-3}$ in all the layers, except for the QDs layer. The QDs layer is modulation doped from the adjacent $n$-type $\operatorname{In}_{0.53} \mathrm{Ga}_{0.35} \mathrm{Al}_{0.12} \mathrm{As}$ barriers.

The band diagram of the prepared heterostructure after the deposition of a $\mathrm{Nb}$ metal on the semiconductor surface is shown in Fig. 1(b). The energy gap of $\mathrm{In}_{0.53} \mathrm{Ga}_{0.47} \mathrm{As}$, $\mathrm{In}_{0.53} \mathrm{Ga}_{0.35} \mathrm{Al}_{0.12} \mathrm{As}$, and $\mathrm{In}_{0.53} \mathrm{Ga}_{0.25} \mathrm{Al}_{0.22} \mathrm{As}$ at low temperature is estimated to be 820,950 , and $1140 \mathrm{meV}$, respectively [34]. At a temperature below $T_{\mathrm{C}}$ of $\mathrm{Nb}$, electrons in $\mathrm{Nb}$ form spin-singlet Cooper pairs. As is clear from Fig. 1(b), the Schottky barrier at the $\mathrm{Nb} / \mathrm{In}_{0.53} \mathrm{Ga}_{0.47} \mathrm{As}$ interface may prevent the penetration of electron Cooper pairs from the $\mathrm{Nb}$ superconductor to the semiconductor heterostructure. The Schottky barrier height is estimated to be $196 \mathrm{meV}$ [35]. The potential barrier is further reduced by $51 \mathrm{meV}$ with the Schottky effect [36]. Then, with the electron concentration of 
$1 \times 10^{18} \mathrm{~cm}^{-3}$ in the $\operatorname{In}_{0.53} \mathrm{Ga}_{0.47}$ As contact layer, the depletion layer width is calculated to be $14.4 \mathrm{~nm}$ for a thick enough $\mathrm{In}_{0.53} \mathrm{Ga}_{0.47} \mathrm{As}$ layer. However, due to the modulation doping from the adjacent $\operatorname{In}_{0.53} \mathrm{Ga}_{0.35} \mathrm{Al}_{0.12} \mathrm{As}$ barrier in Fig. 1(b), the electron concentration in the $\operatorname{In}_{0.53} \mathrm{Ga}_{0.47} \mathrm{As}$ layer is higher, and the depletion layer width is thinner than the $\operatorname{In}_{0.53} \mathrm{Ga}_{0.47} \mathrm{As}$ layer thickness of $10 \mathrm{~nm}$.

The proximity effect, that is, the penetration of electron Cooper pairs to a normal region depends on the coherence length of electron Cooper pairs, and it is calculated to be $9.16 \times 10^{-4}\left(N_{3 \mathrm{D}}\right)^{1 / 3} / T^{1 / 2}[\mathrm{~nm}]$ from the known formula [37] and with the physical parameters for InGaAs [23]. $N_{3 \mathrm{D}}$ is the electron concentration in the normal region (assume to be InGaAs), and for $N_{3 \mathrm{D}}=1 \times 10^{18} \mathrm{~cm}^{-3}$, the coherence length is $916 / T^{1 / 2}[\mathrm{~nm}]$ at $T[\mathrm{~K}] . N_{3 \mathrm{D}}$ will be lower in the above-discussed depletion layer with the thickness of $\sim 10 \mathrm{~nm}$ at the Schottky interface. In this thin depletion layer with the relatively low barrier height, electron wave function penetrates into the depletion layer, and this results in the effective residual electron concentration. Even when it is assumed to be three orders of magnitude lower, that is, $1 \times 10^{15} \mathrm{~cm}^{-3}$, the coherence length is estimated to be $91.6 / T^{1 / 2}[\mathrm{~nm}]$, and this is much longer than the depletion layer width at the $\mathrm{Nb} / \mathrm{In}_{0.53} \mathrm{Ga}_{0.47} \mathrm{As}$ interface. Thus, the penetration of electron Cooper pairs into the InGaAs-based heterostructure becomes possible.

With the modulation doping of the InAs QDs, the depletion layers appear in the $\operatorname{In}_{0.53} \mathrm{Ga}_{0.35} \mathrm{Al}_{0.12} \mathrm{As}$ barriers adjacent to the QDs. The luminescence peak from the InAs QDs is $\sim 790 \mathrm{meV}$, as shown later. When the band offset of $\Delta E_{\mathrm{C}}: \Delta E_{\mathrm{V}}=0.7: 0.3$ is assumed in the InGaAs-based heterostructure [34], the conduction-band effective potential barrier height at the $\operatorname{In}_{0.53} \mathrm{Ga}_{0.35} \mathrm{Al}_{0.12} \mathrm{As} / \mathrm{InAs} \mathrm{QD}$ interface is $112 \mathrm{meV}$. With the $n$-type doping of $1 \times 10^{18} \mathrm{~cm}^{-3}$ in the $\mathrm{In}_{0.53} \mathrm{Ga}_{0.35} \mathrm{Al}_{0.12} \mathrm{As}$ barrier, the depletion layer width is estimated to be $12.7 \mathrm{~nm}$. With the consideration similar to the above Schottky barrier tunneling, it is possible for Cooper pairs to tunnel the low-height barrier coherently through the InAs QDs.

Luminescence from the $\mathrm{Nb}$-superconductor/InGaAs-based semiconductor heterostructure samples can be observed by the optical access from the backside through the InP substrate. Because InP has a high refractive index of $\sim 3.5$, the total internal reflection at an InP/air interface limits the photon extraction efficiency to below $2 \%$ [38]. To prevent this, Nbbased metal-embedded semiconductor pillar structures were fabricated [39]. The details of the fabrication process is given elsewhere [38], but the main processes are as follows: First, pillar arrays were fabricated on the sample surface shown in Fig. 1(a) with electron-beam lithography and the subsequent reactive ion etching (RIE). The pillar height is $\sim 700 \mathrm{~nm}$, and the pillar diameter ranges from 0.2 to $2 \mu \mathrm{m}$. A $200 \mathrm{~nm}$ thick $\mathrm{Nb}$ layer was then deposited on the pillar array surface. The $\mathrm{SC}$ property of the $\mathrm{Nb}$ layer was measured on a simultaneously evaporated reference sample. After evaporation of an additional $\sim 1.5 \mu \mathrm{m}$ thick silver (Ag) layer on the $\mathrm{Nb}$ surface, the sample was turned upside down, and the Ag surface was pasted to a supporting glass substrate with ultraviolet curing resin. Then, the $350 \mu \mathrm{m}$ thick InP substrate was removed with mechanical polishing and the subsequent RIE or selective chemical etching. The finally prepared structure is the array of
InGaAs-based semiconductor pillars embedded in the $\mathrm{Nb} / \mathrm{Ag}$ metal. Although $\mathrm{Nb}$ is not a highly reflecting metal, this metal-embedded structure substantially improves the external collection efficiency of photons emitted from InAs QDs [39].

The prepared sample was set to a liquid-helium cryostat. A continuous-wave He-Ne laser at the wavelength of $632.8 \mathrm{~nm}$ was focused to one of the semiconductor pillars through an objective lens with the numerical aperture of 0.4. Photons emitted from InAs QDs were collected with the same lens, dispersed by a double monochromator with the focal length of $50 \mathrm{~cm}$, and detected with a liquid nitrogen-cooled photodiode array detector.

\section{OPTICAL PROPERTIES BELOW AND ABOVE SC CRITICAL TEMPERATURE}

The SC property of the $\mathrm{Nb}$ layer deposited on the semiconductor surface was measured with the reference sample. The temperature dependence of the differential resistance is shown in Fig. 2(a). The previously reported $T_{\mathrm{C}}$ of $\mathrm{Nb}$ is $9.26 \mathrm{~K}$ [40], and it is estimated to be $9.07 \mathrm{~K}$ in the present $\mathrm{Nb}$ layer. The slight decrease of $T_{\mathrm{C}}$ may be due to slight impurity inclusion during the evaporation, and the gradual increase of the differential resistance up to $9.15 \mathrm{~K}$ suggests that $\mathrm{SC}$ regions remain inhomogeneously up to this temperature.

Figure 2(b) shows the temperature dependence of InAs-QD luminescence spectra measured on one of the pillars with a diameter of $1.5 \mu \mathrm{m}$. The excitation laser power is $20 \mu \mathrm{W}$, and the focused beam diameter is estimated to be $2 \mu \mathrm{m}$, covering the whole pillar surface. This gives an excitation power density of $600 \mathrm{~W} / \mathrm{cm}^{2}$. The luminescence peak is around $790 \mathrm{meV}$ and is slightly blueshifted for the lower temperature. Although the temperature-dependent variation of the luminescence spectra is modest, the peak intensity shows drastic change near $T_{\mathrm{C}}$ of $\mathrm{Nb}$. The temperature dependence of the QD luminescence peak intensity is shown in Fig. 2(c), with an abrupt increase at $T_{\mathrm{C}}$ of the $\mathrm{Nb}$ superconductor and reproduces the luminescence intensity enhancement previously observed in SC LEDs [22,29]. This clear correlation to $T_{\mathrm{C}}$ shows that the phenomenon is closely related to superconductivity.

We worked on similar luminescence measurements on another pillar, and the results are shown in Fig. 3. The spectra were measured at 4 and $10 \mathrm{~K}$, with an excitation power of $600 \mathrm{~W} / \mathrm{cm}^{2}$. The luminescence intensity is much enhanced at $4 \mathrm{~K}$ in comparison to $10 \mathrm{~K}$. This correlation of the luminescence intensity to $T_{\mathrm{C}}$ reproduces that of Fig. 2(c). The distinct feature in Fig. 3 is that we observe a sharp peak and a sharp edge at the photon energy of $\sim 790 \mathrm{meV}$ below $T_{\mathrm{C}}$. It disappears above $T_{\mathrm{C}}$. We previously found that this special feature is very sensitive and easily disappears with a slight temperature rise under the weaker photoexcitation [31]. We also observe luminescence intensity instability in the photon energy range of 790-793 $\mathrm{meV}$ and $801-806 \mathrm{meV}$ below $T_{\mathrm{C}}$. These unusual features observed in the InAs-QD luminescence spectra below $T_{\mathrm{C}}$ manifests the superconductivity effect in the luminescence processes of QDs. We study modeling of the observed phenomena considering the superconductivity effect in radiative recombination processes of QDs in the next section and discuss the relation of the luminescence intensity enhancement and the distinct spectral change below $T_{\mathrm{C}}$. 


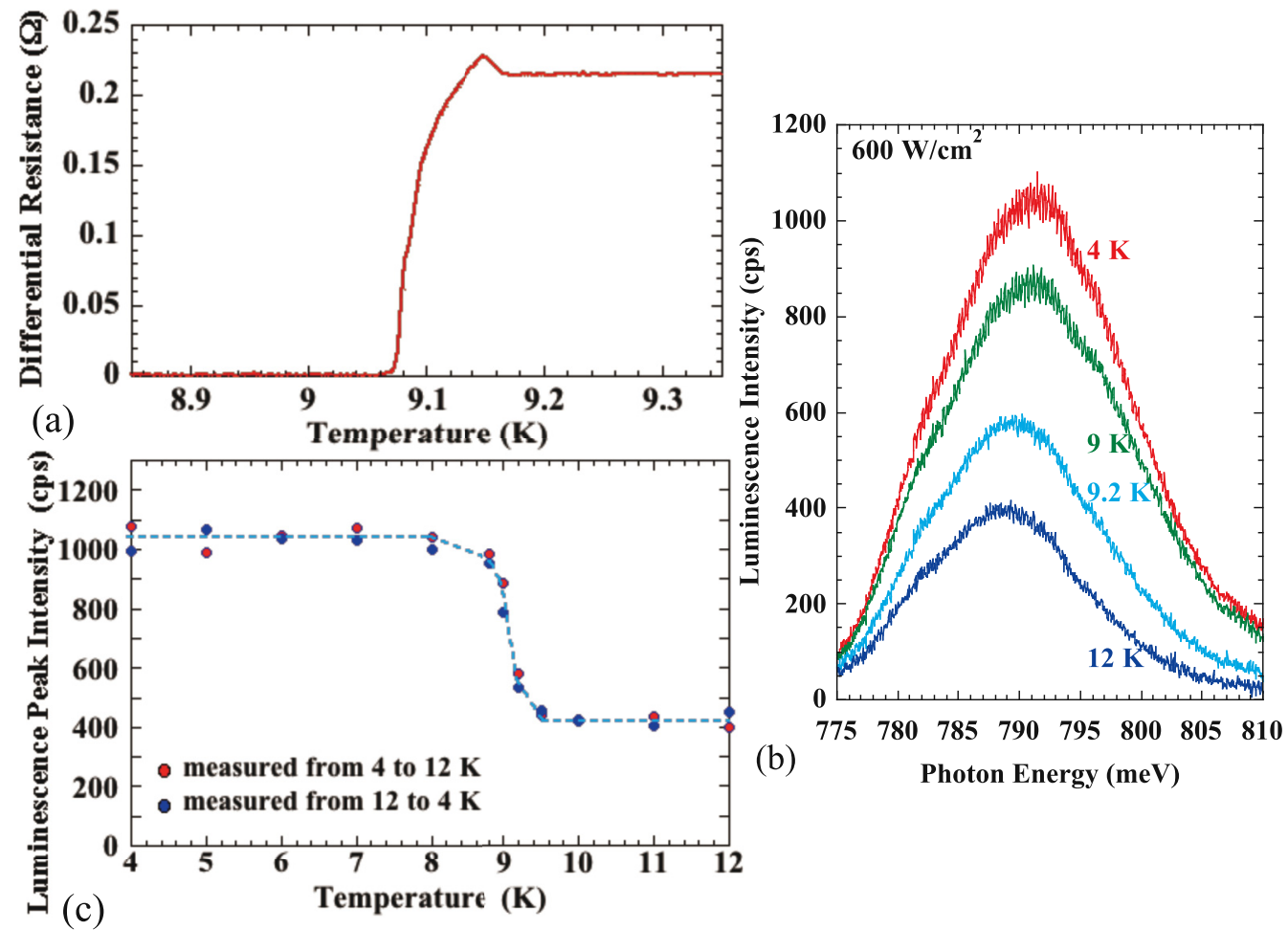

FIG. 2. (Color online) (a) Temperature dependence of differential resistance measured on the deposited Nb layer. (b) Temperature dependence of InAs-QD luminescence spectra. (c) Temperature dependence of QD luminescence peak intensity. To confirm the relation to $T_{\mathrm{C}}=9.07 \mathrm{~K}$ of $\mathrm{Nb}$, measured data for the temperature rising and falling sequences are shown.

\section{MODEL FITTING OF OBSERVED LUMINESCENCE SPECTRA}

As discussed in Sec. I, the proximity effect, especially the opening of SC gap on a normal region surface that is in close proximity to a superconductor, was directly observed with

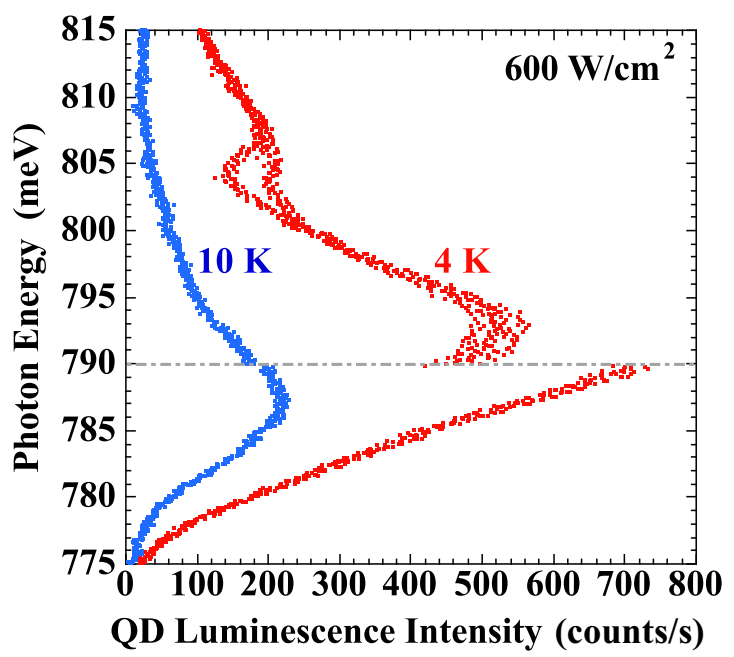

FIG. 3. (Color online) Luminescence spectra observed at 4 and $10 \mathrm{~K}$ with $600 \mathrm{~W} / \mathrm{cm}^{2}$ excitation power from another pillar with the diameter of $1.5 \mu \mathrm{m}$. Only the measured photon counts are plotted without connecting lines. The alternate long and short dashed line is the guide to the eye, indicating the luminescence intensity sharp edge.
STS [25]. It was also observed that SC DOS is broadened with the increase of the distance from the superconductor/normal interface. This broadening was attributed to a finite-lifetime effect of quasiparticles at the SC gap edge state. This effect was included in the Bardeen-Cooper-Schrieffer (BCS) theory by adding an imaginary part to the quasiparticle energy $E$ of the SC DOS [41],

$$
N(E, \Gamma) / N_{0}=\operatorname{Re}\left[\frac{E_{\mathrm{F}}-E+i \Gamma}{\left[\left(E_{\mathrm{F}}-E+i \Gamma\right)^{2}-\Delta^{2}\right]^{1 / 2}}\right],
$$

where $E_{\mathrm{F}}$ is the Fermi energy in the conduction band, $\Delta$ is a half of the SC gap, and $\Gamma$ is the phenomenologically introduced lifetime broadening ( $E<E_{\mathrm{F}}$ is mainly discussed below). $N_{0}$ is the DOS in the normal state. $\operatorname{Re}[]$ is the real part of the physical quantity.

In the measured pillar with the diameter of $1.5 \mu \mathrm{m}$, the QD numbers involved in the luminescence are estimated to be $\sim 600$. The conduction band of the InAs QDs is modulation doped from the neighboring $n$-type $\operatorname{In}_{0.53} \mathrm{Ga}_{0.35} \mathrm{Al}_{0.12} \mathrm{As}$ barriers and is filled with electrons up to the Fermi energy, as schematically shown in Fig. 1(b). Hole population in the valence-band QD lowest-energy states with the external photoexcitation regulates luminescence from the QDs. Considering the estimated large number of QDs, we approximate the energy distribution of the QD lowest-energy states that contribute to the photon emission with a Gaussian function,

$$
G(E)=N_{Q D} \exp \left[-\frac{\left(E-E_{F}-\delta\right)^{2}}{\Delta E^{2}}\right],
$$


where $E$ is the photon energy, $\sqrt{2} \Delta E$ is the half energy width of the Gaussian distribution, and $\delta$ is the energy separation between the ensemble Gaussian peak energy and the Fermi energy $E_{\mathrm{F}}$. $\left(E_{\mathrm{F}}\right.$ in this equation means the energy separation of conduction and valence band energy states of a QD of which conduction-band energy state is in resonance with the electron Fermi energy. In other words, the energy reference is the valence band state energy of that QD.) $N_{\mathrm{QD}}$ is the constant proportional to the total number of luminescent InAs QDs. In general, radiative recombination rate is proportional to the DOS of a relevant medium. Considering the modification of the local DOS with the superconductivity effect, we fit the observed luminescence spectra with the product of Eqs. (1) and (2), i.e., $G(E) N(E, \Gamma)$. The details of the model fitting are described below.

We measured the excitation power dependence of the QD luminescence spectra at $4 \mathrm{~K}$, where the minority hole population in the InAs QDs changes with the excitation level. The measured luminescence spectra are summarized in Fig. 4(a) for 75, 300, and $600 \mathrm{~W} / \mathrm{cm}^{2}$ and Fig. 4(b) for 30 and $150 \mathrm{~W} / \mathrm{cm}^{2}$. The sharp edge observed at $\sim 790 \mathrm{meV}$ becomes sharper for the higher excitation, while it becomes less distinct for the lower excitation. The luminescence intensity instability in the photon-energy range of $790-793 \mathrm{meV}$ is always observed, regardless of the excitation power. The instability in the photon-energy range of $801-806 \mathrm{meV}$ is more distinct for the higher excitation power.

The solid lines in Fig. 4 are the model fitting with Eqs. (1) and (2). For this fitting, we assumed the electron Fermi energy to be $E_{\mathrm{F}}=791.0 \mathrm{meV}$. The SC gap $2 \Delta_{0}$ of $\mathrm{Nb}$ at $0 \mathrm{~K}$ is reported to be $2.91 \pm 0.035 \mathrm{meV}$ [40]. Based on the temperature dependence of the SC gap predicted by the BCS theory [42], a half of the SC gap $\Delta$ is estimated as $1.42 \mathrm{meV}$ at $4 \mathrm{~K}$. The energy range within the alternate long and short dashed lines corresponds to the SC gap. Concerning the empty SC DOS above the SC gap, we assumed no additional enhancement of the QD photon emission originating from the modified DOS. Therefore, the solid line for the energy above $E_{\mathrm{F}}+\Delta$ is calculated with the QD Gaussian function of Eq. (2), and this line is tentatively extended into the SC gap region. It is also not a simple problem how to terminate the fitting with the product of Eqs. (1) and (2) within the SC gap by the consideration of the SC DOS lifetime broadening, and we tentatively terminate the fitting at the middle of the SC gap.

The situation is more complex within the SC gap considering the Cooper-pair tunneling process to each QD state. Cooper-pair transport through a QD state depends on whether a single electron is present or not in the QD state beforehand due to strong Coulomb interactions [43]. Supercurrent carried by Cooper pairs is reversed coherently when a single electron is present in the QD state, and then coherent interference of supercurrent will take place. Population of QD states near the Fermi level varies with time during the luminescent event with the external photoexcitation, and therefore coherent interference of supercurrent is time dependent. The luminescence intensity instability observed in the SC gap reflects this timevarying supercurrent flow to the conduction-band QD states of the InAs QDs. The QD luminescence above the photon energy of $\sim 804 \mathrm{meV}$ originates from the QD excited states. The population instability in the lowest-energy QD states,
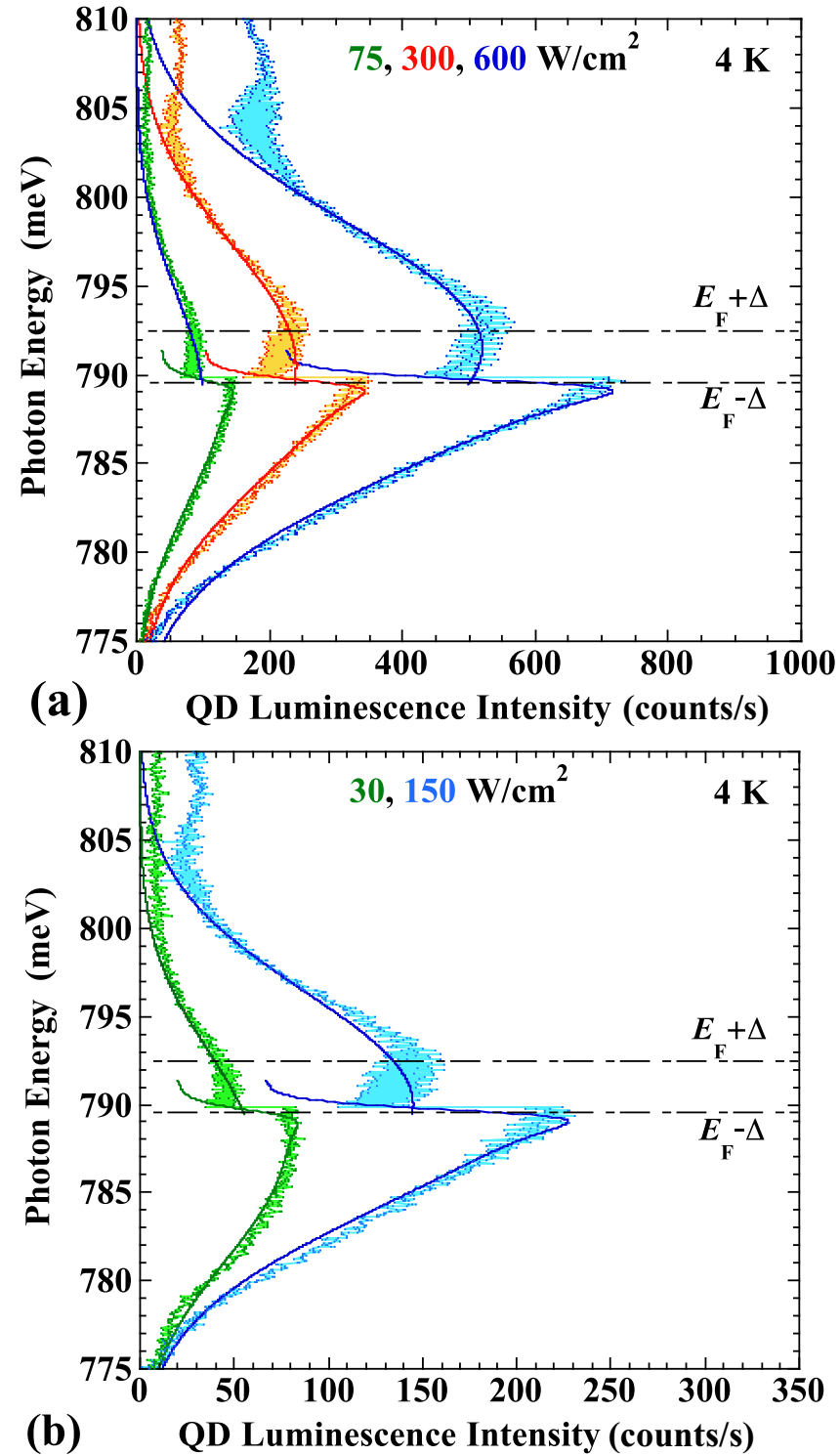

FIG. 4. (Color online) Measured excitation power dependence of QD luminescence spectra. The measured photon counts are connected with neighboring counts with thin lines: (a) 75,300 , and $600 \mathrm{~W} / \mathrm{cm}^{2}$; (b) 30 and $150 \mathrm{~W} / \mathrm{cm}^{2}$. The solid lines are fitting with the parameters of $E_{\mathrm{F}}=791.0 \mathrm{meV}$ and $\Delta=1.42 \mathrm{meV}$.

especially that of minority holes, influences the population of the excited states. Therefore, the luminescence intensity instability observed around the photon energy of $804 \mathrm{meV}$ is related to the induced population instability in the first excited states. Since the population of the excited states increases for the higher photoexcitation, the instability around the photon energy of $804 \mathrm{meV}$ is more distinct for the higher excitation power in Fig. 4.

The SC DOS with the lifetime broadening used for the fitting of the QD luminescence spectra is shown in Fig. 5. The lifetime broadening $\Gamma$ used for the fitting is $0.6 \mathrm{meV}$ for all the measurements shown in Fig. 4. The SC DOS without broadening is also shown as a reference. The Gaussian distribution of the QD energy states, shown in Fig. 5, is the 


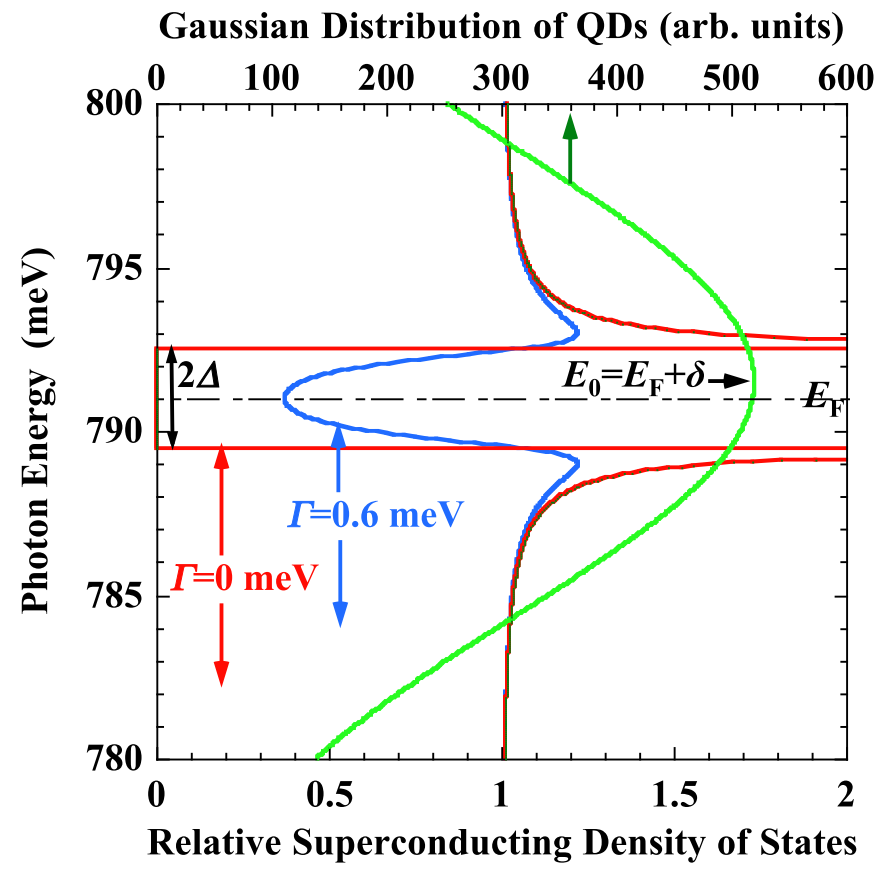

FIG. 5. (Color online) Broadened SC DOS with $\Gamma=0.6 \mathrm{meV}$ and Gaussian distribution of QD energy states employed for the fitting in Fig. 4 are shown. The Gaussian peak is given by $E_{0}=E_{\mathrm{F}}+\delta$, where $E_{\mathrm{F}}$ is the Fermi energy and the energy separation $\delta$ from $E_{\mathrm{F}}$ is given as $0.5 \mathrm{meV}$ for the case of $600 \mathrm{~W} / \mathrm{cm}^{2}$ photoexcitation. SC DOS without broadening $(\Gamma=0 \mathrm{meV})$ is also shown. $2 \Delta$ is the $\mathrm{SC}$ gap.

one used for the fitting of the spectrum observed with the photoexcitation power of $600 \mathrm{~W} / \mathrm{cm}^{2}$ shown in Fig. 4(a). The peak energy of the Gaussian distribution is shifted by $\delta$ from the Fermi energy $E_{\mathrm{F}}$, and $\delta=0.5 \mathrm{meV}$ was used in Fig. 5 . The excitation power dependence of the Gaussian function parameters used for the fitting of each spectrum is shown in Fig. 6. The fitted QD luminescence peak intensity, shown in Fig. 6(a), and the half energy width $\sqrt{2} \Delta E$ shown in Fig. 6(b) naturally increase for the higher excitation power. The fitted QD luminescence peak energy, shown in Fig. 6(b), given by the energy separation $\delta$ from the Fermi energy $E_{\mathrm{F}}$, is blueshifted for the higher excitation power due to the hole population filling in the valence-band QD states. This result clearly shows that the QD luminescence peak is energetically close to the SC gap for the higher excitation in the present sample, and the sharp edge is more clearly observed below $T_{\mathrm{C}}$. On the other hand, the QD luminescence peak is redshifted below the SC gap for the lower excitation, and the effect of the SC DOS on the luminescence spectra becomes milder.

\section{DISCUSSION}

In the previous SC LEDs with QW active layers, we observed luminescence intensity enhancement [22,29] and photon emission rate enhancement $[23,30]$ below $T_{\mathrm{C}}$ of the $\mathrm{Nb}$ SC electrodes, and the observed superconductivity effect was quantitatively well explained with the theory [27]. However, their luminescence spectra showed little variation below and above $T_{\mathrm{C}}$. This is attributed to the continuous QW DOS.
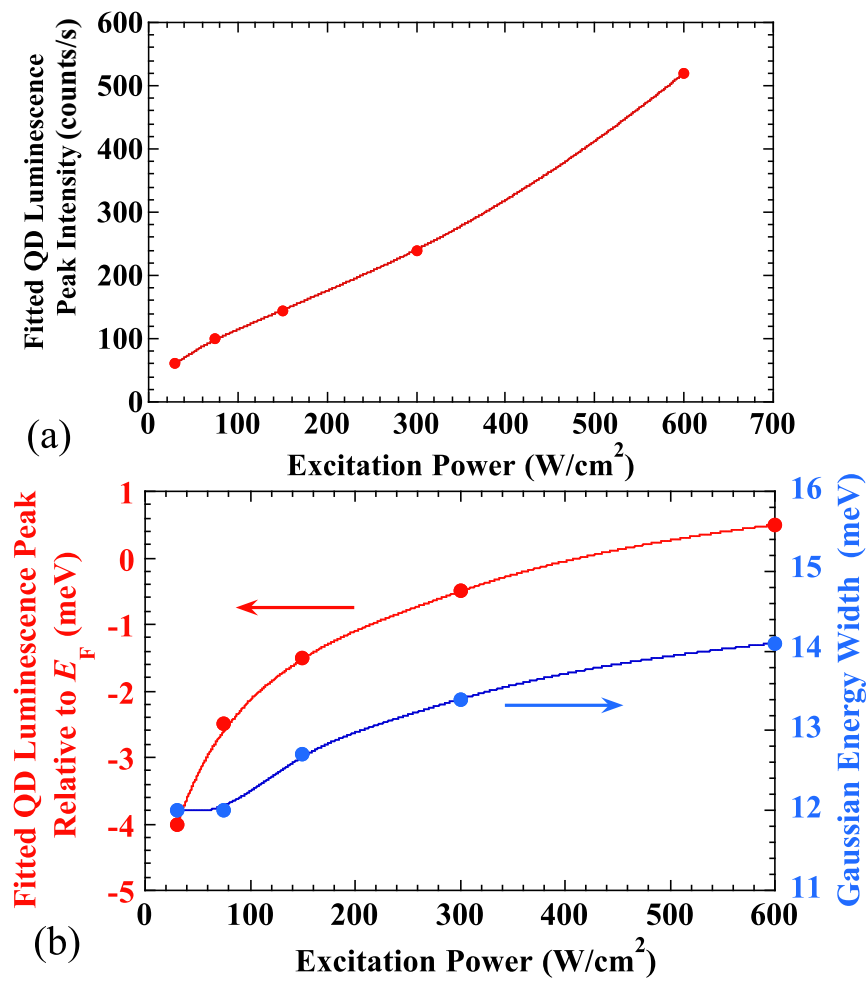

FIG. 6. (Color online) Excitation power dependence of the Gaussian-function parameters used for fitting the spectra, shown in Fig. 4. (a) The QD PL peak intensity. (b) The QD PL peak energy relative to the electron Fermi energy (given by the energy separation $\delta$ ) and Gaussian half energy width.

The ensemble QD luminescence is generally fitted with the Gaussian function (random energy distribution of individually independent QD emission lines), but each QD emission is intrinsically sharp discrete lines. We observed the QD emission line width of $\sim 50 \mu \mathrm{eV}$ from an InAs QD with undoped InGaAlAs barriers grown by MBE in the similar manner on an InP (311)B substrate [44]. Together with the individual sharp QD emission lines, the enhancement of QD luminescence is modified, depending on whether their energies are in resonance or off resonance to the SC DOS, and this results in the sharp edge in the QD luminescence spectra. This sharp edge is averaged out with the continuous DOS in QW structures. The situation changes when the superconductivity effect is assumed both in the conduction and valence bands [45], where the photon energy of the luminescence enhancement is definitely determined by the energy separation of the conduction- and valence-bands SC DOS.

Another important difference is the energy distribution of photoexcited holes. In the case of QDs, excited holes distribute among QDs, depending on their energy relaxation processes, and the Gaussian-broadened ensemble luminescence is generally observed. On the other hand, in doped QWs schematically shown in Fig. 7, excited holes relax to the band edge of the lowest subband. The luminescence related to the Fermi level in the conduction band takes place with the valence-band state with the energy shifted by $\left(m_{\mathrm{e}} / m_{\mathrm{h}}\right) E_{\mathrm{F}}$, where $m_{\mathrm{e}}$ and $m_{\mathrm{h}}$ are the electron and hole effective masses, respectively. Experimentally, the Fermi energy-related luminescence peak 


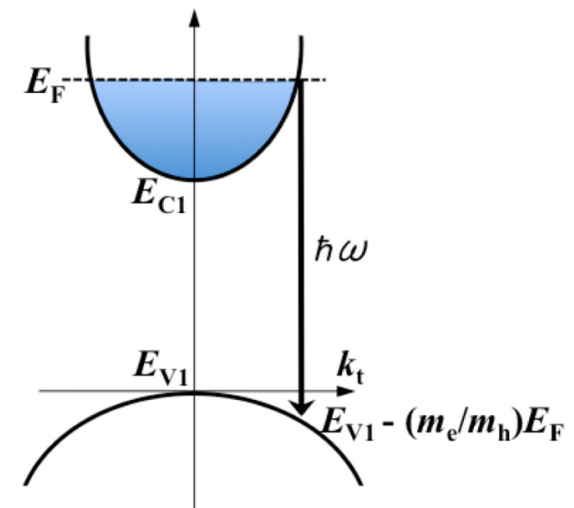

FIG. 7. (Color online) Schematic band diagram of a doped QW. $E_{\mathrm{C} 1}$ and $E_{\mathrm{V} 1}$ are the lowest subband energies in the conduction and valence band, respectively. $\boldsymbol{k}_{\mathrm{t}}$ is the wave vector in the QW plane. The arrow in the downward direction indicates the photon emission with the photon energy of $\hbar \omega$ from the Fermi level to the valence band QW state.

has been observed with a modulation-doped 25 ML thick GaAs/AlGaAs QW at $2 \mathrm{~K}$, and this peak was blueshifted by $81.6 \mathrm{meV}$ from the main luminescence peak [46]. Although the energy shift will be much less in our previous InGaAs QW-based SC LEDs with the lower barrier height [22], this experiment suggests that we have less chance to observe the luminescence spectra related to the SC DOS near the Fermi level with the previous SC LEDs.

Concerning the lifetime broadening of $0.6 \mathrm{meV}$ used for fitting the QD luminescence spectra, the broadening of SC DOS mainly takes place in the $100 \mathrm{~nm}$ thick $\mathrm{In}_{0.53} \mathrm{Ga}_{0.35} \mathrm{Al}_{0.12} \mathrm{As}$ barrier layer. Concerning the broadening mechanisms, we considered the influence of elastic impurity scattering and inelastic scattering, such as electron-phonon and repulsive electron-electron interactions on Cooper-pair luminescence [27]. Recently, we worked on time-resolved measurements of Cooper-pair luminescence from InAs QDs and measured the temperature dependence of the Cooper-pair radiative recombination rate [47]. We could fit the measured Cooper-pair recombination rate enhancement below $T_{\mathrm{C}}$ with our theory employing the broadening parameter of $\Gamma \sim$ $1.2 \mathrm{meV}$. The lifetime broadening may be sample dependent, but this value is reasonably close to the present fitted value of $0.6 \mathrm{meV}$.

Semiconductor QDs frequently exhibit spectral diffusion, that is, time-dependent reversible spectral shift of QD emission lines is observed [48]. This is attributed to the Stark effect caused by fluctuating local electric fields originating from charging and discharging of nearby impurities. Energy shifts up to $3.5 \mathrm{meV}$ have been observed [48]. The spectral diffusion will quench the spectral resonance of QD emission lines to the SC DOS, and change the tunneling processes of Cooper pairs to QD states time dependently, terminating the coherent transport of supercurrent through QD states. This possibly contributes to the effective additional lifetime broadening of SC DOS interacting with QD states.

The comparison of the QD luminescence spectra, shown in Figs. 2(b) and 3, raises the question why the sharp edge is not always observed in the luminescence spectra even

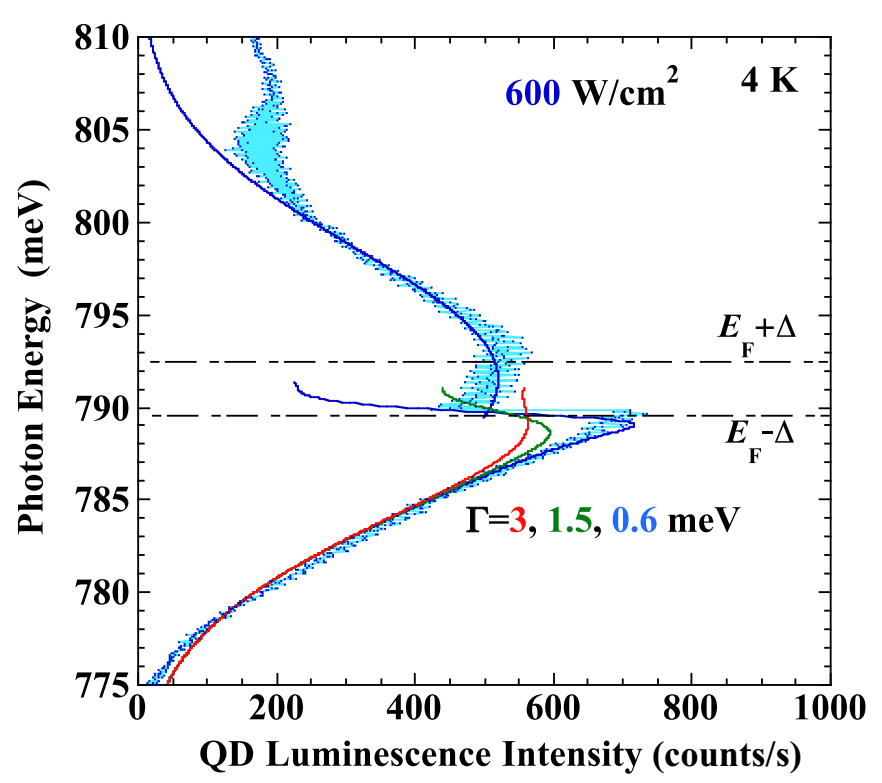

FIG. 8. (Color online) Lifetime-broadening dependence of the fitted luminescence spectra for the one observed with the excitation power of $600 \mathrm{~W} / \mathrm{cm}^{2}$.

from the InAs QDs. The lifetime broadening discussed above is dependent on the local environment around QDs and is dependent on samples. From this viewpoint, we study the lifetime broadening effect on the QD luminescence spectra, and the result is shown in Fig. 8 for the observed spectrum at the excitation power of $600 \mathrm{~W} / \mathrm{cm}^{2}$. With the increase of the broadening parameter $\Gamma$ from $0.6 \mathrm{meV}$, the sharp edge in the luminescence spectrum is diminished. It is almost indistinguishable for $\Gamma$ equal to $3 \mathrm{meV}$. Therefore, we believe that cleaner environment around QDs, which reduces scattering of Cooper pairs and spectral diffusion of QD emission lines, is necessary for the observation of the signature of the SC DOS in the QD luminescence spectra.

The analysis that takes into account effects of Cooper pairing on the recombination process [27] explains the enhancement of luminescence intensity below $T_{\mathrm{C}}$. According to the BCS theory, effects of SC correlation would be energetically limited within an energy range of $E_{\mathrm{F}} \pm \Delta$. On the other hand, the luminescence intensity experimentally found in Figs. 2(b) and 3 is enhanced in the wider energy range than $E_{\mathrm{F}} \pm \Delta$ at low temperature. In our experiments, as discussed above, there are several factors that may broaden the effective energy range. Indeed, we consider a broadening effect due to the distribution in energy levels among QDs through Eq. (2) for fitting the data in Figs. 4 and 8. Such a broadening effect, however, should be explained in more convincing ways beyond the present phenomenological description. This may be an important open issue.

\section{CONCLUSIONS}

We studied luminescence spectra of InAs QDs in an $n$-type InGaAs-based heterostructure, where electron Cooper pairs penetrate from an adjacent $\mathrm{Nb}$ superconductor with the proximity effect. We observed substantial luminescence 
intensity enhancement and a sharp edge in luminescence spectra of InAs QDs below the SC critical temperature of $\mathrm{Nb}$.

We explained the observed sharp edge in the luminescence spectra by considering opening of SC gap and modification of DOS near the electron Fermi level in the $n$-type semiconductor heterostructure. We showed that observation of the sharp edge luminescence spectra is sensitive to the quasiparticle lifetime broadening. Our finding open a way to study the proximity effect of superconductivity with optical technique and offers the possibility to find new physics related to superconductivity and optoelectronics.

\section{ACKNOWLEDGMENTS}

The authors are grateful to T. Akazaki for his arrangement of our collaborative research. This paper is supported in part by Nano-Macro Materials, Devices and Systems Research Alliance, and Hokkaido Innovation through NanoTechnology Support (HINTS).
[1] J. L. O'Brien, A. Furusawa, and J. Vuckovic, Nat. Photon. 3, 687 (2009).

[2] N. Gisin, G. Ribordy, W. Tittel, and H. Zbinden, Rev. Mod. Phys. 74, 145 (2002).

[3] C. H. Bennett, G. Brassard, C. Crepeau, R. Jozsa, A. Peres, and W. K. Wootters, Phys. Rev. Lett. 70, 1895 (1993).

[4] D. Bouwmeester, J.-W. Pan, K. Mattle, M. Eibl, H. Weinfurter, and A. Zeilinger, Nature (London) 390, 575 (1997).

[5] I. Marcikic, H. de Riedmatten, W. Tittel, H. Zbinden, and N. Gisin, Nature (London) 421, 509 (2003).

[6] S. Bose, V. Vedral, and P. L. Knight, Phys. Rev. A 57, 822 (1998).

[7] J.-W. Pan, D. Bouwmeester, H. Weinfurter, and A. Zeilinger, Phys. Rev. Lett. 80, 3891 (1998).

[8] H. de Riedmatten, I. Marcikic, J. A. W. van Houwelingen, W. Tittel, H. Zbinden, and N. Gisin, Phys. Rev. A 71, 050302 (2005).

[9] P. G. Kwiat, K. Mattle, H. Weinfurter, A. Zeilinger, A. V. Sergienko, and Y. Shih, Phys. Rev. Lett. 75, 4337 (1995).

[10] R. Jin, R. Shimizu, I. Morohashi, K. Wakui, M. Takeoka, S. Izumi, T. Sakamoto, M. Fujiwara, T. Yamashita, S. Miki, H. Terai, Z. Wang, and M. Sasaki, Sci. Rep. 4, 7468 (2014).

[11] C. L. Salter, R. M. Stevenson, I. Farrer, C. A. Nicoll, D. A. Ritchie, and A. J. Shields, Nature (London) 465, 594 (2010).

[12] A. Dousse, J. Suffczynski, A. Beveratos, O. Krebs, A. Lemaitre, I. Sagnes, J. Bloch, P. Voisin, and P. Senellart, Nature (London) 466, 217 (2010).

[13] M. Mueller, S. Bounouari, K. D. Joens, M. Glaessl, and P. Michler, Nat. Photon. 8, 224 (2014).

[14] W. J. Munro, K. Nemoto, and A. G. White, J. Mod. Opt. 48, 1239 (2001).

[15] R. J. Young, R. M. Stevenson, A. J. Hudson, C. A. Nicoll, D. A. Ritchie, and A. J. Shields, Phys. Rev. Lett. 102, 030406 (2009).

[16] T. Kuroda, T. Mano, N. Ha, H. Nakajima, H. Kumano, B. Urbaszek, M. Jo, M. Abbarchi, Y. Sakuma, K. Sakoda, I. Suemune, X. Marie, and T. Amand, Phys. Rev. B 88, 041306(R) (2013).

[17] A. J. Hudson, R. M. Stevenson, A. J. Bennett, R. J. Young, C. A. Nicoll, P. Atkinson, K. Cooper, D. A. Ritchie, and A. J. Shields, Phys. Rev. Lett. 99, 266802 (2007).

[18] G. Breit and E. Teller, Astrophys. J. 91, 215 (1940).

[19] A. Hayat, P. Ginzburg, and M. Orenstein, Nat. Photon. 2, 238 (2008).

[20] L. Hofstetter, S. Csonka, J. Nygard, and C. Schoenenberger, Nature (London) 461, 960 (2009).

[21] I. Suemune, T. Akazaki, K. Tanaka, M. Jo, K. Uesugi, M. Endo, H. Kumano, E. Hanamura, H. Takayanagi, M. Yamanishi, and H. Kan, Jpn. J. Appl. Phys. 45, 9264 (2006).
[22] Y. Hayashi, K. Tanaka, T. Akazaki, M. Jo, H. Kumano, and I. Suemune, Appl. Phys. Express 1, 011701 (2008).

[23] H. Sasakura, S. Kuramitsu, Y. Hayashi, K. Tanaka, T. Akazaki, E. Hanamura, R. Inoue, H. Takayanagi, Y. Asano, C. Hermannstadter, H. Kumano, and I. Suemune, Phys. Rev. Lett. 107, 157403 (2011).

[24] P. G. De Gennes, Rev. Mod. Phys. 36, 225 (1964).

[25] L. Serrier-Garcia, J. C. Cuevas, T. Cren, C. Brun, V. Cherkez, F. Debontridder, D. Fokin, F. S. Bergeret, and D. Roditchev, Phys. Rev. Lett. 110, 157003 (2013).

[26] W. Chang, S. M. Albrecht, T. S. Jespersen, F. Kuemmeth, P. Krogstrup, J. Nygård, and C. M. Marcus, Nat. Nanotechnol. 10, $232(2015)$

[27] Y. Asano, I. Suemune, H. Takayanagi, and E. Hanamura, Phys. Rev. Lett. 103, 187001 (2009).

[28] J. Unterhinninghofen, D. Manske, and A. Knorr, Phys. Rev. B 77, 180509(R) (2008).

[29] I. Suemune, Y. Hayashi, S. Kuramitsu, K. Tanaka, T. Akazaki, H. Sasakura, R. Inoue, H. Takayanagi, Y. Asano, E. Hanamura, S. Odashima, and H. Kumano, Appl. Phys. Express 3, 054001 (2010).

[30] I. Suemune, H. Sasakura, Y. Asano, H. Kumano, R. Inoue, K. Tanaka, T. Akazaki, and H. Takayanagi, IEICE Electron. Express 9, 1184 (2012).

[31] S. S. Mou, H. Irie, Y. Asano, K. Akahane, H. Kurosawa, H. Nakajima, H. Kumano, M. Sasaki, and I. Suemune, IEEE J. Sel. Top. Quantum Electron. 21, 7900111 (2015).

[32] N. A. Jahan, C. Hermannstaedter, J-H. Huh, H. Sasakura, T. J. Rotter, P. Ahirwar, G. Balakrishnan, K. Akahane, M. Sasaki, H. Kumano, and I. Suemune, J. Appl. Phys. 113, 033506 (2013).

[33] K. Akahane and N. Yamamoto, J. Cryst. Growth 378, 450 (2013).

[34] I. Vurgaftman, J. R. Meyer, and L. R. Ram-Mohan, J. Appl. Phys. 89, 5815 (2001).

[35] K. Kajiyama, Y. Mizushima, and S. Sakata, Appl. Phys. Lett. 23, 458 (1973).

[36] S. M. Sze, Physics of Semiconductor Devices, 2nd ed. (Wiley, New York, 1981).

[37] T. Akazaki, T. Kawakami, and J. Nitta, Appl. Phys. Lett. 66, 6121 (1989).

[38] I. Suemune, H. Nakajima, X. Liu, S. Odashima, T. Asano, H. Iijima, J.-H. Huh, Y. Idutsu, H. Sasakura, and H. Kumano, Nanotechnology 24, 455205 (2013).

[39] H. Kumano, H. Nakajima, S. Ekuni, Y. Idutsu, H. Sasakura, and I. Suemune, Adv. Math. Phys. 2010, 1 (2010). 
[40] V. Novotny and P. P. M. Meincke, J. Low Temp. Phys. 18, 147 (1975).

[41] R. C. Dynes, V. Narayanamurti, and J. P. Garno, Phys. Rev. Lett. 41, 1509 (1978).

[42] M. Tinkham, Introduction to Superconductivity, 2nd ed. (Dover, New York, 2004).

[43] J. A. van Dam, Y. V. Nazarov, E. P. A. M. Bakkers, S. De Franceschi, and L. P. Kouwenhoven, Nature (London) 442, 667 (2006).
[44] X. Liu, K. Akahane, N. A. Jahan, N. Kobayashi, M. Sasaki, H. Kumano, and I. Suemune, Appl. Phys. Lett. 103, 061114 (2013).

[45] P. Hlobil and P. P. Orth, Phys. Rev. B 91, 205303 (2015).

[46] M. Bugajski and K. Reginski, Opto-Electron. Rev. 4, 84 (1996).

[47] S. S. Mou, H. Irie, Y. Asano, K. Akahane, H. Nakajima, H. Kumano, M. Sasaki, A. Murayama, and I. Suemune, (unpublished).

[48] J. Seufert, R. Weigand, G. Bacher, T. Kuemmell, A. Forchel, K. Leonardi, and D. Hommel, Appl. Phys. Lett. 76, 1872 (2000). 